\title{
Maximum likelihood estimation of linkage between a marker gene and a quantitative trait locus. II. Application to backcross and doubled haploid populations
}

\author{
Z. W. LUO* \& M. J. KEARSEY \\ School of Biological Sciences, University of Birmingham, Birmingham B15 2TT, UK
}

\begin{abstract}
The algorithm for estimating both the recombination fraction between a marker gene and a locus affecting a quantitative trait, and also the means and variances of the QTL genotypes, is extended to backcross and doubled haploid populations. The simulation experiments show that estimates of these parameters can be obtained with acceptable accuracy and results are compared with those obtained using $\mathrm{F}_{2}$ populations studied previously (Luo \& Kearsey, 1989).
\end{abstract}

Keywords: doubled haploid populations, linkage, marker gene, quantitative trait.

\section{Introduction}

Since the publication of the paper by Botstein et al. (1980), we have witnessed growing interest in the use of molecular genetic markers to locate unknown genes, particularly those genetic factors associated with quantitative variation. These approaches are all developed from a variety of methods examined by biometric geneticists in the last 40 years.

Three aspects of the use of markers have attracted the interest of biometricians and geneticists. Firstly, the detection of polygenes (or QTLs) with associated developments of statistical approaches appropriate to various breeding experiments (Thoday, 1961; Jayakar, 1970; Hill, 1975; Lander \& Botstein, 1989 and Luo, 1989); secondly, measurement of the statistical power of different experimental designs for polygene detection (McMillan \& Robertson, 1974; Soller \& Brody, 1976; Soller \& Genizi, 1978; Luo \& Kearsey, 1989]; finally, estimation of linkage between a given marker gene and a putative QTL. Jayakar (1970) developed formulae to estimate marker-QTL recombination fractions in two different designs for use in studying natural populations, but they were unrealistic in practice through lack of appropriate estimates of environmental

*Present address: Department of Zoology, University of Edinburgh, West Mains Road, Edinburgh EH9 3JT, UK. variance. Weller (1986) applied maximum likelihood techniques to the analysis of the $\mathrm{F}_{2}$ generation of a cross between two inbred lines in order to estimate not only the recombination fraction between a marker locus and a QTL but also the nature and size of the effect. Because the numerical algorithm employed by Weller to search the likelihood surface was based on an unreasonable assumption, i.e. the parameters to be estimated in the likelihood function were independent of each other, his algorithm could not confirm that the estimates obtained were, in fact, the maximum likelihood estimates. In an attempt to overcome this problem we described, in a previous paper (Luo \& Kearsey, 1989), a maximum likelihood approach for estimating the recombination fraction in a segregating population $\left(\mathrm{F}_{2}\right)$ between a marker gene and a QTL as well as estimating the means and variances of the three genotypes of the QTL. In this paper, we extend the algorithm to two other experimental designs, i.e. backcrosses and doubled haploids.

\section{Theoretical approach}

We consider a breeding programme starting with two inbred lines, one of which is homozygous for the alleles $M_{1}$ and $Q_{1}$ for the marker and QTL respectively, while the other is homozygous for the alleles $\mathrm{M}_{2}$ and $\mathrm{Q}_{2}$. The marker alleles are assumed to be co-dominant, and the 
recombination fraction between the two loci is denoted by $r$. The $F_{1}$ is either crossed to the parent with genotype $\mathrm{M}_{2} \mathrm{M}_{2} \mathrm{Q}_{2} \mathrm{Q}_{2}$ to produce a backcross generation with family size of $n_{\mathrm{b}}$ or used to generate $n_{\mathrm{d}}$, doubled haploid families (e.g. by anther culture). For simplicity, we further assume that all the families have a constant size of $n$. The means and variances of the quantitative trait among the marker genotypes are as shown in Table 1.

\section{Development of the analytical method}

Since the linkage between marker and QTL is incomplete, the individuals within each of the two possible marker genotypes are a mixture of two quantitative trait genotypes, i.e. $Q_{1} Q_{2}$ and $Q_{2} Q_{2}$ for the backcross design but $\mathrm{Q}_{1} \mathrm{Q}_{1}$ and $\mathrm{Q}_{2} \mathrm{Q}_{2}$ for the doubled haploids. Let $\mu_{11}, \mu_{12}$ and $\mu_{22}$ represent the means and $\sigma_{11}^{2}$, $\sigma_{12}^{2}$ and $\sigma_{22}^{2}$ represent the variances of the three genotypes for the quantitiative trait. The variances include not only environmental variation but also genetic variation at other loci affecting the quantitative trait. Therefore, the means of the marker classes can be partitioned into the following:

for the backcross,

$X_{12}=(1-r) \mu_{12}+r \mu_{22}$

$X_{22}=r \mu_{12}+(1-r) \mu_{22}$

and for the doubled haploids,

$X_{11}=(1-r) \mu_{11}+r \mu_{22}$

$X_{22}=r \mu_{11}+(1-r) \mu_{22}$

while the variances of the marker groups can be partitioned as

$S_{12}^{2}=(1-r)\left[\left(\mu_{12}-X_{12}\right)^{2}+\sigma_{12}^{2}\right]+r\left[\left(\mu_{22}-X_{12}\right)^{2}+\sigma_{22}^{2}\right]$

$S_{22}^{2}=r\left[\left(\mu_{12}-X_{22}\right)^{2}+\sigma_{12}^{2}\right]+(1-r)\left[\left(\mu_{22}-X_{22}\right)^{2}+\sigma_{22}^{2}\right]$

Table 1 Basic statistics of the marker genotypes in (a) the backcross and (b) the doubled haploid

Statistics of the

quantitative trait

\begin{tabular}{llll} 
quantitative trait & $M_{1} M_{1}$ & $M_{1} M_{2}$ & $M_{2} M_{2}$ \\
\hline Means & $X_{11}$ & $X_{12}$ & $X_{22}$ \\
Variances & $S_{11}^{2}$ & $S_{12}^{2}$ & $S_{22}^{2}$ \\
Sample size & & & \\
$\quad$ (a) & 0 & $n_{1}$ & $n_{2}$ \\
(b) & $n_{1}$ & 0 & $n_{2}$ \\
\hline
\end{tabular}

for the backcross, and as

$S_{11}^{2}=(1-r)\left[\left(\mu_{11}-X_{11}\right)^{2}+\sigma_{11}^{2}\right]+r\left[\left(\mu_{22}-X_{11}\right)^{2}+\sigma_{22}^{2}\right]$

$S_{22}^{2}=r\left[\left(\mu_{11}-X_{22}\right)^{2}+\sigma_{11}^{2}\right]+(1-r)\left[\left(\mu_{22}-X_{22}\right)^{2}+\sigma_{22}^{2}\right]$

for the doubled haploids.

The composite distribution densities of the relevant marker classes can be written as (Hasselblad, 1966; Day, 1969)

$f_{M_{12}}(x)=(1-r) f_{12}(x)+r f_{22}(x)$

$f_{M_{22}}(x)=r f_{12}(x)+(1-r) f_{22}(x)$

for the backcross design; and as

$f_{M_{11}}(x)=(1-r) f_{11}(x)+r f_{22}(x)$

$f_{M_{22}}(x)=r f_{11}(x)+(1-r) f_{22}(x)$

for the doubled haploids, where

$f_{i j}(x)=\frac{1}{\sqrt{2 \pi \sigma_{i j}^{2}}} \exp \left[-\frac{\left(x-\mu_{i j}\right)^{2}}{\sigma_{i j}^{2}}\right]$

and

$i, j=1,2$.

Therefore the likelihood function can be written as

$L_{\mathrm{B}}\left(\mu_{12}, \mu_{22}, \sigma_{12}^{2}, \sigma_{22}^{2}, r\right)=\left[\prod_{i=1}^{n_{1}} f_{M_{12}}\left(x_{i}\right)\right]\left[\prod_{j=1}^{n_{2}} f_{M_{22}}\left(x_{j}\right)\right]$

for the entire backcross generation, and as

$L_{\mathrm{D}}\left(\mu_{11}, \mu_{22}, \sigma_{11}^{2}, \sigma_{22}^{2}, r\right)=\left[\prod_{i=1}^{n_{1}} f_{M_{13}}\left(x_{i}\right)\right]\left[\prod_{j=1}^{n_{2}} f_{M_{22}}\left(x_{j}\right)\right]$

for the entire doubled haploid population.

The logarithm functions of (5) will be, respectively,

$$
\begin{aligned}
L_{\mathrm{B}}(r)= & \sum_{i=1}^{n_{1}} \ln f_{M_{12}}\left(x_{i}\right)+\sum_{j=1}^{n_{2}} \ln f_{M_{22}}\left(x_{j}\right)=\ln \frac{1}{\sqrt{2 \pi}}\left(n_{1}+n_{2}\right) \\
& +\sum_{i=1}^{n_{1}} \ln \left\{\frac{1-r}{\sigma_{12}} \exp \left[-\frac{\left(x_{i}-\mu_{12}\right)^{2}}{2 \sigma_{12}^{2}}\right]\right. \\
& \left.+\frac{r}{\sigma_{22}} \exp \left[-\frac{\left(x_{i}-\mu_{22}\right)^{2}}{2 \sigma_{22}^{2}}\right]\right\} \\
& +\sum_{j=1}^{n_{2}} \ln \left\{\frac{r}{\sigma_{12}} \exp \left[-\frac{\left(x_{j}-\mu_{12}\right)^{2}}{2 \sigma_{12}^{2}}\right]\right. \\
& \left.+\frac{1-r}{\sigma_{22}} \exp \left[-\frac{\left(x_{j}-\mu_{22}\right)^{2}}{2 \sigma_{22}^{2}}\right]\right\}
\end{aligned}
$$


and

$$
\begin{aligned}
L_{\mathrm{D}}(r)= & \sum_{i=1}^{n_{1}} \ln f_{M_{11}}\left(x_{i}\right)+\sum_{j=1}^{n_{2}} \ln f_{M_{22}}\left(x_{j}\right)=\ln \frac{1}{\sqrt{2 \pi}}\left(n_{1}+n_{2}\right) \\
& +\sum_{i=1}^{n_{1}} \ln \left\{\frac{1-r}{\sigma_{11}} \exp \left[-\frac{\left(x_{i}-\mu_{11}\right)^{2}}{2 \sigma_{11}^{2}}\right]\right. \\
& \left.+\frac{r}{\sigma_{22}} \exp \left[-\frac{\left(x_{i}-\mu_{22}\right)^{2}}{2 \sigma_{22}^{2}}\right]\right\} \\
& +\sum_{j=1}^{n_{2}} \ln \left\{\frac{r}{\sigma_{11}} \exp \left[-\frac{\left(x_{j}-\mu_{11}\right)^{2}}{2 \sigma_{11}^{2}}\right]\right. \\
& \left.+\frac{1-r}{\sigma_{22}} \exp \left[-\frac{\left(x_{j}-\mu_{22}\right)^{2}}{2 \sigma_{22}^{2}}\right]\right\} .
\end{aligned}
$$

Equation $6(\mathrm{a}$ and $\mathrm{b})$ include the other four unknown parameters besides $r$. However, following the method used to analyse the $F_{2}$ generation, equations (1a) and (2a) provide the following solutions to the unknowns in (6a)

$$
\begin{aligned}
& \mu_{22}=\frac{1}{1-2 r}\left[(1-r) X_{M_{12}}-r X_{M_{22}}\right] \\
& \mu_{12}=\left\{X_{M_{12}}+X_{M_{22}}\right\}-\mu_{22}
\end{aligned}
$$

and

$\sigma_{22}^{2}=\frac{1}{1-2 r}\left[(1-r) e_{2}-r e_{1}\right]$

$\sigma_{12}^{2}=e_{1}+e_{2}-\sigma_{22}^{2}$

where

$e_{1}=S_{12}^{2}-\left[(1-r)\left(\mu_{12}-X_{12}\right)^{2}+r\left(\mu_{22}-X_{12}\right)^{2}\right]$

$e_{2}=S_{22}^{2}-\left[r\left(\mu_{12}-X_{22}\right)^{2}+(1-r)\left(\mu_{22}-X_{22}\right)^{2}\right]$

while equations $(1 \mathrm{~b})$ and $(2 \mathrm{~b})$ yield the corresponding solutions to the means and variances of the QTL genotypes in the doubled haploid design (6b)

$$
\begin{aligned}
& \mu_{22}=\frac{1}{1-2 r}\left[(1-r) X_{M_{22}}-r X_{M_{11}}\right] \\
& \mu_{11}=\left\{X_{M_{11}}+X_{M_{22}}\right\}-\mu_{22}
\end{aligned}
$$

and

$$
\begin{aligned}
& \sigma_{22}^{2}=\frac{1}{1-2 r}\left[(1-r) e_{2}-r e_{1}\right] \\
& \sigma_{11}^{2}=e_{1}+e_{2}-\sigma_{22}^{2}
\end{aligned}
$$

where

$$
\begin{aligned}
& e_{1}=S_{11}^{2}-\left[(1-r)\left(\mu_{11}-X_{11}\right)^{2}+r\left(\mu_{22}-X_{11}\right)^{2}\right] \\
& e_{2}=S_{22}^{2}-\left[r\left(\mu_{11}-X_{22}\right)^{2}+(1-r)\left(\mu_{22}-X_{22}\right)^{2}\right] .
\end{aligned}
$$

When these estimates of the means $\left(\mu_{i j}\right)$ and variances $\left(\sigma_{i j}^{2}\right)$ obtained from equations (7) or from $(8)$ are incorporated into the log likelihood functions (6a) and (6b) for these two designs, respectively, then these functions will involve only one unknown parameter, the recombination fraction $r$. Searching these functions numerically for their maximum values with respect to $r$, will yield the maximum likelihood estimates of $r$ for each design. Since the means and variances of the quantitative trait have been expressed as the monotonic functions about $r$, according to the invariant property of the maximum likelihood estimator (Mood et al., 1974), i.e. if $\hat{\theta}$ is the maximum likelihood estimate of $\theta$ in the distribution density $f\left(x_{j} ; \theta\right)$ and $\tau(\cdot)$ is a transformation of the parameter space $\Theta$, then a maximum likelihood estimate of $\tau(\theta)$ is $\tau(\hat{\theta})$. Thus formulae (7) and (8) directly give maximum likelihood estimates of the means and variances of the quantitative trait in the two different designs.

Furthermore, the two QTL genotypes, i.e. $Q_{1} Q_{2}$ and $Q_{2} Q_{2}$, in the backcross generation and the two corresponding genotypes, i.e. $Q_{1} Q_{2}$ and $Q_{2} Q_{2}$, in the doubled haploid population, should have the same environmental variances. We can therefore use the following

$\sigma_{\mathrm{b}}^{2}=\frac{n_{1}}{N} \sigma_{12}^{2}+\frac{n_{2}}{N} \sigma_{22}^{2}$

and

$\sigma_{\mathrm{d}}^{2}=\frac{n_{1}}{N} \sigma_{11}^{2}+\frac{n_{2}}{N} \sigma_{22}^{2}$

to approximate the environmental variances for the two populations respectively, where $N$ represents the total experimental size of backcross design but represents the number of families for the doubled haploids.

\section{Description of the algorithm}

In the last section, it has been demonstrated that the likelihood functions $\left[\mathrm{L}_{\mathrm{B}}(\mathrm{r})\right.$ or $\left.\mathrm{L}_{\mathrm{D}}(\mathrm{r})\right]$ involve only one unknown parameter $r$. This greatly simplifies the algorithm to search the likelihood surface for the relevant maximum likelihood estimates. In our previous paper (Luo \& Kearsey, 1989), an iterative algorithm was described to obtain the estimates from $F_{2}$ populations. The same numerical algorithm will be employed here. 


\section{Simulation of the two designs and data analysis}

Two FORTRAN-77 computer programs were designed both to simulate the genetic behaviour of linkage between a marker locus and a QTL and to work out the required maximum likelihood estimates folowing the algorithms described in the previous discussion. The principle of the simulation of genetic behaviour of the marker-QTL linkage is described in detail elsewhere (Luo, 1989; Luo \& Kearsey, 1989).

All possible combinations of three recombination fractions between the marker gene and the QTL $(r=0.15,0.25,0.35)$, two narrow heritabilities $\left(h_{n}^{2}=0.1,0.5\right)$ and three dominance ratios $(d r=0.0$, $0.5,1.0$ ) for the QTL (these genetic parameters are defined by using an $\mathrm{F}_{2}$ population as a standard) were simulated for both designs with fixed size of 500 while each of the 500 doubled haploid families has a size of 5 . These are the same parameter combinations used for the $F_{2}$ generation by Luo \& Kearsey (1989).

\section{Results}

The maximum likelihood estimates of the recombination fraction between the marker locus and the QTL and the corresponding standard errors, given a sample size of 500, are recorded in Table 2 for the doubled haploid population and in Table 3 for the backcross generation. The maximum likelihood estimates of the means of the two possible genotypes at the QTL can be found, together with their standard errors, in Tables 4 and 5 for the doubled haploids and the backcross generation, respectively. The maximum likelihood estimates of the environmental variances and their standard errors for the two designs are given in Table 6.

It is clear from Table 2 that there is no significant difference between the maximum likelihood estimates of the recombination fraction obtained from the data of means of doubled haploid families and their true values. The standard errors of these estimates consistently decrease as the heritability of the QTL increases, but neither the dominance ratio of the QTL nor the true values of the estimated parameter has any obvious influence on the estimates or their standard errors.

Table 3 shows that the recombination fractions can also be estimated adequately by use of the backcross data. However, when the heritability is low, these fractions are slightly underestimated at its true value of 0.15 but are slightly overestimated when the true value is equal to or higher than 0.25 . The standard errors of

Table 2 The maximum likelihood estimates of the recombination fraction between the marker and QTL, where $h_{n}^{2}$ and $d r$ represent narrow heritability and dominance ratio of the QTL respectively (doubled haploid families)

\begin{tabular}{lllll}
\hline \multicolumn{5}{l}{ True recombination fraction } \\
\cline { 3 - 5 }$h_{n}^{2}$ & $d r$ & 0.15 & 0.25 & 0.35 \\
\hline 0.1 & 0.0 & $0.1800 \pm 0.0458$ & $0.2856 \pm 0.0374$ & $0.3564 \pm 0.0447$ \\
0.1 & 0.5 & $0.1218 \pm 0.0316$ & $0.2880 \pm 0.0469$ & $0.3742 \pm 0.0648$ \\
0.1 & 1.0 & $0.1731 \pm 0.0490$ & $0.2849 \pm 0.0469$ & $0.3821 \pm 0.0400$ \\
0.5 & 0.0 & $0.1550 \pm 0.0200$ & $0.2666 \pm 0.0332$ & $0.3603 \pm 0.0316$ \\
0.5 & 0.5 & $0.1470 \pm 0.0224$ & $0.2581 \pm 0.0374$ & $0.3559 \pm 0.0300$ \\
0.5 & 1.0 & $0.1561 \pm 0.0245$ & $0.2427 \pm 0.0265$ & $0.3750 \pm 0.0283$ \\
\hline
\end{tabular}

Table 3 The maximum likelihood estimates of the recombination fraction between the marker and QTL, where $h_{n}^{2}$ and $d r$ represent narrow heritability and dominance ratio of the QTL respectively (backcross generation)

\begin{tabular}{lllll}
\hline \multicolumn{5}{c}{ True recombination fraction } \\
\cline { 3 - 5 }$h_{n}^{2}$ & $d r$ & 0.15 & 0.25 & 0.35 \\
\hline 0.1 & 0.0 & $0.1287 \pm 0.0700$ & $0.2742 \pm 0.0557$ & $0.3807 \pm 0.0500$ \\
0.1 & 0.5 & $0.1105 \pm 0.0721$ & $0.2796 \pm 0.0529$ & $0.3720 \pm 0.0633$ \\
0.1 & 1.0 & $0.0854 \pm 0.0656$ & $0.2817 \pm 0.0510$ & $0.3720 \pm 0.0633$ \\
0.5 & 0.0 & $0.1644 \pm 0.0361$ & $0.2588 \pm 0.0480$ & $0.3690 \pm 0.0400$ \\
0.5 & 0.5 & $0.1554 \pm 0.0361$ & $0.2627 \pm 0.0353$ & $0.5392 \pm 0.0300$ \\
0.5 & 1.0 & $0.1575 \pm 0.0332$ & $0.2554 \pm 0.0374$ & $0.3428 \pm 0.0283$ \\
\hline
\end{tabular}


Table 4 The maximum likelihood estimates and expected values of the means of the two QTL genotypes for different genetic situations, where $h_{n}^{2}, d r, \mu$ and $r$ are narrow heritability, dominance ratio, expected means of the QTL genotypes and true recombination fraction between the genetic marker and QTL respectively (doubled haploid families)

\begin{tabular}{|c|c|c|c|c|c|}
\hline \multirow[b]{2}{*}{$d r$} & \multirow[b]{2}{*}{$r$} & \multicolumn{2}{|l|}{$h_{n}^{2}=0.1$} & \multicolumn{2}{|l|}{$h_{n}^{2}=0.5$} \\
\hline & & $\hat{\mu}_{11}$ & $\hat{\mu}_{22}$ & $\hat{\mu}_{11}$ & $\hat{\mu}_{22}$ \\
\hline \multirow[t]{3}{*}{0.0} & 0.15 & $109.63 \pm 1.1091$ & $99.31 \pm 1.0488$ & $119.87 \pm 0.6633$ & $100.08 \pm 0.5568$ \\
\hline & 0.25 & $109.63 \pm 0.8775$ & $98.68 \pm 1.0392$ & $115.85 \pm 5.3768$ & $100.17 \pm 0.9434$ \\
\hline & 0.35 & $109.23 \pm 1.0100$ & $99.70 \pm 1.0247$ & $116.45 \pm 5.2900$ & $99.97 \pm 1.3266$ \\
\hline$\mu$ & & 108.94 & 100.00 & 120.00 & 100.00 \\
\hline \multirow{3}{*}{0.5} & 0.15 & $109.13 \pm 2.5436$ & $99.91 \pm 1.0198$ & $118.94 \pm 0.5745$ & $100.09 \pm 0.4583$ \\
\hline & 0.25 & $109.76 \pm 1.2042$ & $99.20 \pm 1.1533$ & $117.19 \pm 3.3601$ & $100.05 \pm 0.5658$ \\
\hline & 0.35 & $109.16 \pm 1.4071$ & $99.58 \pm 0.8485$ & $116.08 \pm 5.0665$ & $100.23 \pm 0.9434$ \\
\hline$\mu$ & & 108.44 & 100.00 & 118.86 & 100.00 \\
\hline \multirow[t]{3}{*}{1.0} & 0.15 & $110.39 \pm 2.1111^{* *}$ & $99.60 \pm 0.7000$ & $117.01 \pm 0.6083$ & $100.33 \pm 0.5000$ \\
\hline & 0.25 & $109.98 \pm 1.0440^{* *}$ & $99.16 \pm 1.0583$ & $118.64 \pm 3.2655$ & $100.25 \pm 0.3873$ \\
\hline & 0.35 & $109.14 \pm 1.1091$ & $99.61 \pm 0.8889$ & $119.03 \pm 2.8160$ & $99.75 \pm 0.9185$ \\
\hline$\mu$ & & 107.30 & 100.00 & 116.32 & 100.00 \\
\hline
\end{tabular}

Table 5 The maximum likelihood estimates and expected values of the means of the two QTL genotypes for different genetic situations, where $h_{n}^{2} d r, \mu$ and $r$ are narrow heritability, dominance ratio, expected means of the QTL genotypes and true recombination fraction between the genetic marker and QTL respectively (backcross generation)

\begin{tabular}{|c|c|c|c|c|c|}
\hline \multirow[b]{2}{*}{$d r$} & \multirow[b]{2}{*}{$r$} & \multicolumn{2}{|l|}{$h_{n}^{2}=0.1$} & \multicolumn{2}{|l|}{$h_{n}^{2}=0.5$} \\
\hline & & $\hat{\mu}_{12}$ & $\hat{\mu}_{22}$ & $\hat{\mu}_{12}$ & $\hat{\mu}_{22}$ \\
\hline \multirow[t]{3}{*}{0.0} & 0.15 & $102.80 \pm 0.9434^{* *}$ & $99.52 \pm 0.9274$ & $109.91 \pm 0.5657$ & $99.89 \pm 0.5657$ \\
\hline & 0.25 & $101.06 \pm 0.9899^{* *}$ & $99.45 \pm 1.6462$ & $110.41 \pm 0.5292$ & $99.65 \pm 0.3000$ \\
\hline & 0.35 & $106.61 \pm 2.3706$ & $98.77 \pm 2.3706$ & $110.14 \pm 0.7280$ & $99.77 \pm 0.7746$ \\
\hline$\mu$ & & 104.47 & 100.00 & 110.00 & 100.00 \\
\hline \multirow[t]{3}{*}{0.5} & 0.15 & $106.02 \pm 1.0149$ & $100.17 \pm 0.7348$ & $114.27 \pm 0.5385$ & $100.06 \pm 0.6481$ \\
\hline & 0.25 & $107.40 \pm 1.1446$ & $98.96 \pm 1.1402$ & $113.26 \pm 1.6817$ & $100.01 \pm 0.8718$ \\
\hline & 0.35 & $107.64 \pm 1.2000$ & $98.75 \pm 1.3000$ & $113.84 \pm 0.9381$ & $100.34 \pm 0.9747$ \\
\hline$\mu$ & & 106.33 & 100.00 & 114.14 & 100.00 \\
\hline \multirow[t]{3}{*}{1.0} & 0.15 & $112.52 \pm 3.4380$ & $100.68 \pm 0.6083$ & $111.24 \pm 0.5385$ & $99.93 \pm 0.4243$ \\
\hline & 0.25 & $105.81 \pm 1.4213$ & $99.02 \pm 1.2961$ & $111.09 \pm 0.6557$ & $99.97 \pm 0.6403$ \\
\hline & 0.35 & $106.17 \pm 1.6026$ & $99.14 \pm 1.3528$ & $110.73 \pm 1.3038$ & $100.41 \pm 0.7211$ \\
\hline$\mu$ & & 107.30 & 100.00 & 116.32 & 100.00 \\
\hline
\end{tabular}

the estimates obtained from this design are mostly higher than those obtained from doubled haploid family means, suggesting that using the doubled haploid family means can yield a more accurate estimate of the recombination fraction for a fixed sample size. Of course each family mean of the doubled haploids with respect to the quantitative trait was based on five individuals.

The phenotypic means of the two genotypes of the QTL are denoted by $\mu_{11}$ and $\mu_{22}$ in the doubled haploid population and by $\mu_{12}$ and $\mu_{22}$ in the backcross generation. The maximum likelihood estimates of these means are listed in Tables 4 and 5 for the two designs. It is clear that these estimates do not deviate significantly from their expected values when the quantitative trait has a high heritability $\left(h_{n}^{2}=0.5\right)$. Moreover, these means can also be estimated with a moderate heritability $\left(h_{n}^{2}=0.1\right)$. The effect of the level of dominance of the increasing allele at the QTL on the accuracy of these estimates differs in the two designs. As can be seen from Tables 4 and 5, increasing dominance makes the expected values of $\mu_{11}$ and $\mu_{22}$ more alike but $\mu_{11}$ and $\mu_{12}$ more unlike. The effect of dominance on the doubled haploids is an artefact of the model since, with 
Table 6 The maximum likelihood estimates of the environmental variances associated with the QTL, where $h_{n}^{2}, d r$ EVE are narrow heritability, dominance ratio and expected variance of the quantitative trait for doubled haploids and backcrosses

\begin{tabular}{|c|c|c|c|c|c|}
\hline \multirow[b]{2}{*}{$h_{n}^{2}$} & \multirow[b]{2}{*}{$d r$} & \multirow[b]{2}{*}{ EVE } & \multicolumn{3}{|c|}{ True recombination fraction } \\
\hline & & & 0.15 & 0.25 & 0.35 \\
\hline \multicolumn{6}{|c|}{ Doubled haploids } \\
\hline 0.1 & 0.0 & 90.00 & $79.73 \pm 9.0500$ & $75.19 \pm 12.2593$ & $83.44 \pm 9.2952$ \\
\hline 0.1 & 0.5 & 90.00 & $87.87 \pm 8.7178$ & $72.72 \pm 10.2387$ & $81.00 \pm 10.7624$ \\
\hline 0.1 & 1.0 & 90.00 & $77.83 \pm 11.3309$ & $78.44 \pm 8.7304$ & $79.52 \pm 12.3778$ \\
\hline 0.5 & 0.0 & 50.00 & $49.20 \pm 4.2661$ & $65.20 \pm 10.2401$ & $61.64 \pm 10.6300$ \\
\hline 0.5 & 0.5 & 50.00 & $49.25 \pm 3.6810$ & $54.83 \pm 4.6750$ & $60.82 \pm 11.3978$ \\
\hline 0.5 & 1.0 & 50.00 & $47.95 \pm 3.3985$ & $53.42 \pm 7.7188$ & $49.10 \pm 6.1237$ \\
\hline \multicolumn{6}{|c|}{ Backcrosses } \\
\hline 0.1 & 0.0 & 90.00 & $79.79 \pm 5.8915$ & $82.79 \pm 5.7879$ & $73.82 \pm 14.1421$ \\
\hline 0.1 & 0.5 & 90.00 & $85.04 \pm 11.4873$ & $75.20 \pm 9.9600$ & $72.92 \pm 9.7783$ \\
\hline 0.1 & 1.0 & 90.00 & $72.53 \pm 4.3336^{* *}$ & $80.20 \pm 7.9869$ & $76.08 \pm 10.9316$ \\
\hline 0.5 & 0.0 & 50.00 & $46.70 \pm 3.9370$ & $44.44 \pm 5.3413$ & $46.48 \pm 6.6588$ \\
\hline 0.5 & 0.5 & 50.00 & $48.37 \pm 3.6986$ & $52.19 \pm 7.5050$ & $49.88 \pm 8.6742$ \\
\hline 0.5 & 1.0 & 50.00 & $46.03 \pm 3.4395$ & $48.82 \pm 4.3070$ & $50.54 \pm 7.6551$ \\
\hline
\end{tabular}

fixed phenotypic variance and heritability, increasing dominance effectively reduces additivity. As a result, with dominance the means are estimated less well in the doubled haploids but better with the backcrosses. The reverse is true with no dominance. The standard errors of the estimates also decline with increasing heritability for both designs.

The maximum likelihood estimates of the environmental variance, $\sigma^{2}$, do not differ significantly from their true values in the doubled haploid families and only one of the 18 situations simulated in the backcross design.

In terms of their abilities to provide accurate estimates of marker-QTL linkage, as well as the phenotypic means and variances of the QTL genotypes, neither of the two designs is consistantly superior to the other over all possible genetic backgrounds of the QTL. The above results do, however, suggest that at low heritabilities the doubled haploid families would be more powerful than the backcross design when the dominance ratio is low.

\section{Discussion}

This paper has concentrated on the use of maximum likelihood techniques to estimate the marker-QTL recombination fraction and the relevant genetic and environmental effects of the QTL. The results obtained from the simulation experiments with 20 replications of each of 18 different combinations of genetic parameters indicate that recombination fractions could be well estimated using these designs. In fact, none of the estimates differed significantly from their expected values for all combinations of genetic parameters considered with experimental size of 500 . However, some significantly biased estimates were observed in the $\mathrm{F}_{2}$ generation design for both the heritabilities simulated (Luo \& Kearsey, 1989).

Accurate estimates of the means and variances of the two QTL genotypes were regularly found from both the designs and the frequency of significantly biased estimates was again lower than that in the $F_{2}$ experiments. The maximum likelihood estimates of the environmental variances associated with the QTL were rarely observed to be different from their actual values in the simulated backcross and doubled haploid experiments, while biased estimates for this parameter were commonly observed in the simulated $\mathrm{F}_{2}$.

The estimation problem, discussed here and in our previous work, is statistically equivalent to resolving a single mixed normal distribution into a few component distributions with common variance. As we have noted in the previous discussion, if the putative QTL is linked with the co-dominant marker gene, the phenotypic distribution of the quantitative trait in each marker class will be an unequal component mixture of two component normal distributions for the backcross and doubled haploids, but of three component normal 
distributions for the $F_{2}$ population. The power of any algorithm leading to a solution to the relevant parameters of a composite distribution depends on the absolute difference between the means of the component distribution for a fixed common variance. For a given heritability, it is obvious that the absolute difference between the means of the homozygous and heterozygous genotypes (e.g. two of the three component distributions) at the QTL in the $F_{2}$ generation is much less than that between the two means of the two possible genotypes (e.g. two component distributions) at the same QTL in either the backcross or the doubled haploid family designs. This would explain why the latter two designs always exhibit higher efficiency in producing the relevant estimates than the $F_{2}$ generation design. In the present studies, the heritability of the quantitative trait plays an important role in determining the mean difference and variance of the component distributions, i.e. the higher the heritability the larger the difference between the means, the smaller the variance and therefore the more efficient the algorithm. Furthermore, it is interesting to note that the markerQTL linkage estimates obtained from the $\mathrm{F}_{2}$ generation regularly have smaller standard errors than those yielded by the other two designs; i.e. the $F_{2}$ data supply more information about linkage for a fixed experimental size (Mather, 1936; 1938).

So far, there have been three basic approaches developed respectively by Hasselblad (1966), Bhattacharya (1967) and Cohen (1967) to carry out dissection of mixed distributions. As a specific genetic application to the problem of separating the mixed distributions, the algorithms developed in the present studies have used the genetic characteristics to provide useful information. This has effectively simplified the estimation procedure and in turn increased the efficiency of the algorithm. Firstly, the proportions of the component distributions were completely determined by the recombination fraction between the marker gene and the linked QTL. Secondly, if the phenotypic means and variances of the recombinant genotypes, estimated from the experimental sample, were used as unbiased estimates of the corresponding population parameters of the composite distributions, then the means and variances of the relevant genotypes at the QTL were uniquely determined by the recombination fraction and these estimates. This results in the maximum likelihood function involving only one unknown parameter, i.e. the recombination fraction. The maximum likelihood estimates of the remaining parameters, including the means and environmental variances of the relevant QTL genotypes, could easily be obtained directly from their functional relationships to the means and variances of the marker genotypes, thus avoiding use of the complicated iterative algorithm suggested by previous authors. In fact, the present algorithms obviously show higher efficiency than the general methods. Hasselblad (1966) claimed that it was extremely difficult to dissect three subdistributions by use of his maximum likelihood algorithm, which was considered by Tan \& Chang (1972) to be the best of the three approaches mentioned before, when the means were separated by less than 2 standard deviation units even with an experimental size of 1,000 . However, using the algorithms developed in this and our previous paper (Luo \& Kearsey, 1989), the component distributions were regularly wellestimated even though the means were separated by much less than one standard deviation, for example $h_{n}^{2}=0.1$ with an experimental size of 500 .

In the previous discussion, we have assumed normality of the distributions of the phenotypes among the marker groups. This assumption is at odds with the fact that incomplete linkage between the marker and the QTL will result in skewness in these marker groups. This assumption may be a source of bias in the estimates of the basic parameters.

\section{References}

BHATTACHARYA, G. G. 1967. A simple method of resolution of a distribution into Gaussian components. Biometrics, 23, 115-135.

BOTSTEIN, D., WHITE, R. L., SKOLNICK, M. AND DAVIES, R. w. 1980. Construction of a genetic linkage map in man using restriction fragment length polymorphism. Am. J. Hum. Genet., 32, 314-331.

COHEN, A. C. 1967. Estimation in mixtures of two normal distributions. Technometrics, 9, 15-28.

DAY, N. E. 1969. Estimating the components of a mixture of normal distributions. Biometrika, 56, 463-474.

HASSElBlad, F. 1966. Mixture of normal distributions. Technometrics, 8, 231-250.

HILL, A. P. 1975. Quantitative linkage: a statistical procedure for its detection and estimation. Ann. Hum. Genet. London, 38, 439-449.

JAYAKAR, S. D. 1970. On the detection and estimation of linkage between a locus influencing a quantitative character and a marker locus. Biometrics, 26, 451-464.

LANDER, E. S. AND BOTSTEIN, D. 1989. Mapping Mendelian factors underlying quantitative traits using RFLP linkage maps. Genetics, 121, 185-199.

Luo, z. w. 1989. Ph.D. Thesis. University of Birmingham.

LUO, Z. W. AND KEARSEY, M. J. 1989. Maximum likelihood estimation of linkage between a marker gene and a quantitative locus. Heredity, 63, 401-408.

MATHER, K. 1936. Types of linkage data and their value. Ann. Eugenics, 7, 251-264.

MATHER, K. 1938. The Measurement of Linkage in Heredity. Methuen and Co. Ltd, London. 
MCMILLAN, I. AND ROBERTSON, A. 1974. The power of methods for the detection of major genes affecting quantitative characters. Heredity, 32, 349-356.

MOOD, A. M., GRAYBILL, F. A. AND BOES, D. C. 1974. Introduction to the Theory of Statistics. McGraw-Hill Book Company, New York.

SOLLER, M. AND BRODY, T. 1976. On the power of experimental designs for the detection of linkage between marker loci and quantitative loci in crosses between inbred lines. Theor. Appl. Genet., 47, 35-39.

SOLLER, M. AND GENIZI, A. 1978. The efficiency of experimental designs for the detection of linkage between a marker locus and a locus affecting a quantitative trait in segregating populations. Biometrics, 34, 47-55.

TAN, W. Y. AND CHANG, w. C. 1972. Some comparisons of the method of moments and the method of maximum likelihood in estimating parameters of a mixture of two normal densities. J. Am. Stat. Assoc., 67, 702-708.

THODAY, J. M. 1961. Location of polygenes. Nature, 191, 368-370.

WELLER, J. I. 1986. Maximum likelihood techniques for the mapping and analysis of quantitative trait loci with the aid of genetic markers. Biometrics, 42, 627-640. 